\title{
Finned Space Radiator Performance Analysis Using Computational Methods
}

\author{
Kevin E. de Conde ${ }^{1}$, Rafael Dias Vilela ${ }^{2}$, Ezio C. Garcia ${ }^{1}$ \\ ${ }^{1}$ Instituto Tecnológico de Aeronáutica (ITA) \\ Praça Marechal-do-Ar Eduardo Gomes, 50 - Vila das Acácias, São José dos Campos, CEP 12228-900, São Paulo, Brazil \\ kevin@ita.br; ezio@ita.br \\ ${ }^{2}$ Centro Universitário de Adamantina (UniFAI) \\ Rua Nove de Julho, 730 - Centro, Adamantina, CEP 17800-800, São Paulo, Brazil \\ rdiasvilela@yahoo.com.br
}

\begin{abstract}
Satellites are typically subjected to large temperature variations due to its orbit, whereas some satellite's equipment sharp temperature limits must be observed in order not to jeopardize satellite's mission. Designed to operate in passive satellites' thermal control, space radiators can be a valuable asset, rejecting heat generated by electronic components and minimizing absorption of heat from external sources (especially the Sun). The present work presents a numerical methodology for performing the analysis of space radiators, and a comparison between a plane radiator and finned radiator in critical operating conditions is presented. The governing equations have been discretized in a two-dimensional grid using a finite volume scheme and the resulting system was solved using Thomas Algorithm in an iterative process, concatenated in two algorithms. The results obtained show the temperature profiles for both radiators studied and a performance analysis is performed, showing the best geometrical configuration for the finned radiator.
\end{abstract}

Keywords: Computational heat transfer, Radiative heat transfer, Radiator performance analysis, Satellite thermal control

\section{Introduction}

In-orbit artificial satellites are subject to large temperature gradients, which are characterized by high temperatures when direct exposed to solar radiation, and by low temperatures when in eclipse. In addition to receiving the incoming Sun radiation and Earth's albedo, satellites have electric and electronic components that generate heat and sensitive components, such as batteries, which need special attention for their narrow temperature range $\left(-5\right.$ to $\left.25^{\circ} \mathrm{C}\right)$. Consequently, satellite thermal design is a very important step in mission design and a space radiator can constitute an important feature in the passive thermal control, such as the SCD-1 Brazilian satellite [1]. Passive thermal control is usually preferred due to the lower cost, lower energy consumption, and higher reliability, especially in cases where the satellite's mission is for a long period [3].

\section{Background}

The spectral distribution of extraterrestrial solar irradiation approximates that of a blackbody at $5800 \mathrm{~K}$, and approximately $98 \%$ of its radiation are within 0 and $3 \mu \mathrm{m}$ (solar band) spectrum. Space radiators, on the other hand, operating at temperatures below $400 \mathrm{~K}$, emit $99.8 \%$ of their radiation in the infrared spectrum (which wavelength is greater than $3 \mu \mathrm{m}$ ). So, space radiators predominantly absorb radiation in the solar band and emit in the infrared band [4]. Consequently, it is common that a space radiator receives an optical treatment in order to lower its absorptivity in the solar band and increase its emissivity in the infrared band. Gilmore suggests that the absorptivity in the solar band should be less than 0.2 and the emissivity in the infrared band exceeds 0.8 [5].

Some studies about space radiators are presented in the literature. Cuco et al. have developed a finned radiator with variable emissivity, which allows reducing the dependence for electric heaters. According to their study, the increased mass, which will elevate the costs, is compensated by the electrical energy saving. This energy saving is of great importance to projects of this kind, once it is a limited resource [6, 7]. In the work by Lieblein and Diedrich, the authors conducted an analysis on materials and geometries used in the construction of space radiators in order to evaluate the relation between mass, ease of manufacture and heat transfer rate provided by the device. They have concluded that the requirements outlined above are usually in conflict [7]. Garcia presented a one-dimensional numerical model that analyses the multimode heat 
transfer in a radiator with rectangular fins as shown in Fig. 1. The study shows the good performance of the radiator, the temperature profile in the base set flip, radiation, and heat loss through radiation. [8]. Silva and Garcia studied the transverse conductivity of an aluminium honeycomb panel for satellites. Using the software SINDA/FLUINT, the numerical results were compared with analytical and experimental results for the numerical model validation and optimization [9]. Kumar et al. proposed a space radiator number-of-fins-based optimization with rectangular fins and the consequent increase of the weight of the radiator. The authors present the results obtained in terms of heat transfer rate for various numbers of flaps built with three different materials (aluminium, steel and brass) [10].

\section{Mathematical Model and Boundary Conditions}

Given the high cost of performing experiments on orbit [1], numerical simulation is valuable and cost-effective alternative. The present work develops a mathematical model for the space radiator proposed by Garcia [8] in a 2-D Cartesian mesh using a finite volume scheme. The aim here is to study the radiator's behavior under critical operation conditions, called operation in the hot and cold cases. The analysis of the hot case considers the period with lowest exposure to eclipse and the highest incidence of solar radiation and the Earth's albedo. In the cold case, the satellite remains a longer under the eclipse thus causing a decrease in incidence solar and Earth's albedo radiation [2].

Considering the space radiator shown in Fig. 1 with flat rectangular fins equally spaced and with the same length and thickness, it is possible to treat the problem using the fact that the analysis for one set of fins (as shown in Fig. 2) can be extended for the whole radiator, with the boundary fins not considered.

The surface arrangement is characterized by the presence of a symmetry axis. It corresponds to the fact that the radiosities leaving surfaces $S_{4}$ and $S_{2}$ reach surface $S_{3}$ with the same intensity. In addition, the net rate of radiation exchanged between $S_{2}$ and $S_{4}$ is zero. The radiosities from $S_{2}, S_{3}$ and $S_{4}$ that reach space can be computed by adding the hypothetical surface $S_{6}$ (see Fig. 2).

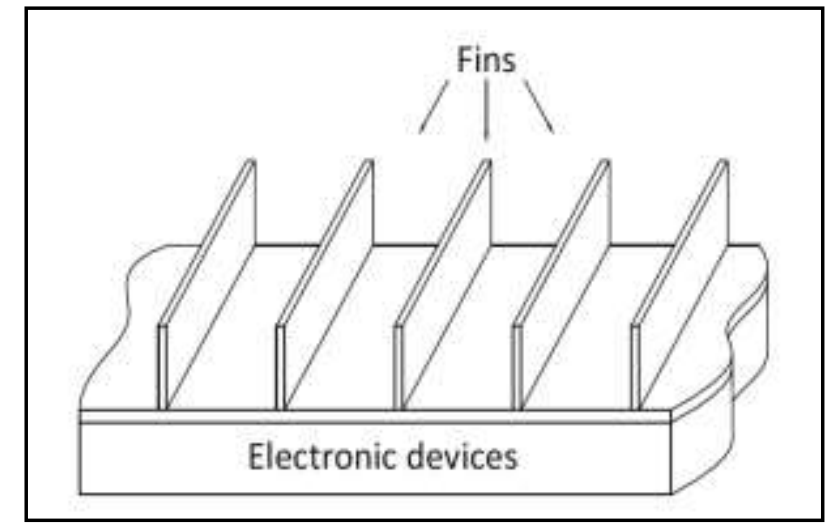

Fig. 1: Finned space radiator proposed by Garcia (1996) [8].

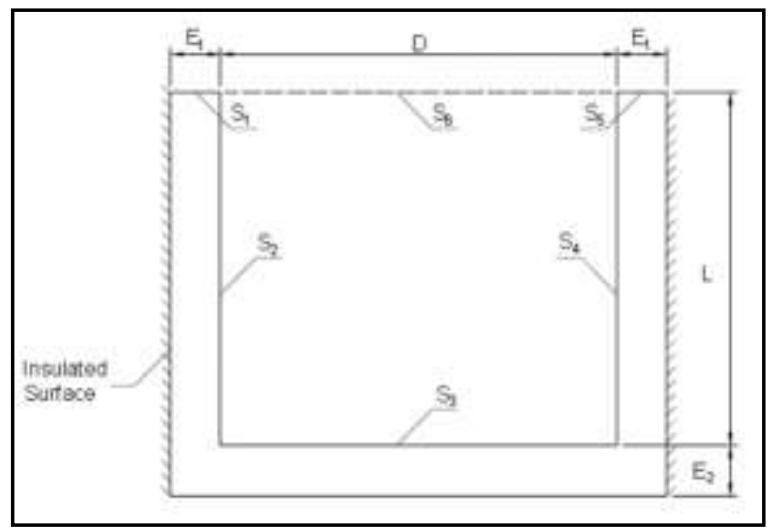

Fig. 2: Geometric parameters and nomenclature.

The present study considers also the following assumptions.

1. Solar radiation focused perpendicularly to the base of the radiator, surface $S_{3}$

2. Constant and temperature-independent thermo-optical properties

3. Uniform properties

4. Two-dimensional conduction heat transfer

5. Steady state

6. No presence of heat generation

7. Isotropic Material

8. Unit depth to surfaces the surfaces of Fig. 2

9. Adoption of the band model (solar and infrared)

10. Independent spectral emissivity and spectral reflectivity of $\lambda$ on the two bands (gray surfaces) 
11. Plate and opaque surfaces $(\rho=1-\varepsilon)$

12. Diffuse surfaces

13. $\alpha=\varepsilon$ for each band (solar and infra-red)

14. Prescribed heat flux from the satellite and the bottom surface of the radiator (see Fig. 4)

15. Negligible boundary effect.

The parameters of the radiator in the study are those proposed by Garcia [8] and are presented in Table 1.

Table 1: Dimensions, material properties and numerical tolerance used in the numerical solution.

\begin{tabular}{cccc}
\hline \hline $\mathrm{N}^{\circ}$ of points & 600 & Tolerance & $1.0 \times 10^{-8}$ \\
$L(m)$ & 0.01 & $\varepsilon_{S I}$ & 0.2 \\
$D(m)$ & 0.01 & $\varepsilon_{S 2}$ & 0.2 \\
$E_{I}(m)$ & 0.005 & $\varepsilon_{I I}$ & 0.8 \\
$E_{2}(m)$ & 0.005 & $\varepsilon_{I 2}$ & 0.8 \\
$k(W / m K)$ & 200 & & \\
\hline \hline
\end{tabular}

Table 2 presents the boundary conditions of heat flux emanating from the electronic devices (see Fig. 1) and solar incidence of hot and cold cases.

The conservation equations have been applied to the physical model and, with the considerations shown above, resulted in a system of integral-partial differential equations, which constitute a set of equations of elliptic mathematical type. Therefore, the equations have been integrated using an essentially centered (i.e. with no artificial dissipation) finite volume scheme, therefore resulting in a system of linear algebraic equations, which has been solved using Thomas' algorithm. An iterative process performed in parallel were used to calculate the temperature profile in the base of the radiator.

Table 2: Conditions of heat flux and solar incidence for both cases.

\begin{tabular}{ccc}
\hline \hline Cases & Hot case & Cold case \\
$q_{E D}^{\prime \prime}\left(\mathrm{W} / \mathrm{m}^{2}\right)$ & 324 & 324 \\
$H_{S u n}\left(\mathrm{~W} / \mathrm{m}^{2}\right)$ & 1,400 & 0 \\
\hline \hline
\end{tabular}

In Fig. 3 it is presented the computational domain, which corresponds to half of the geometric domain shown in Fig. 2, with a mesh, and boundary conditions.

In order to present the discretizing method, we begin by considering the conservation equations. Fourier's law under the presented considerations takes the form:

$$
\frac{\partial}{\partial x}\left(k \frac{\partial T}{\partial x}\right)+\frac{\partial}{\partial y}\left(k \frac{\partial T}{\partial y}\right)=0
$$

Discretizing equation (1) according to the finite volume scheme proposed by Gasparini, we obtain [11]

$$
\begin{aligned}
& k A\left[\frac{T(x+1, y)-T(x, y)}{\delta x}\right]-k A\left[\frac{T(x, y)-T(x-1, y)}{\delta x}\right]+ \\
& k A\left[\frac{T(x, y+1)-T(x, y)}{\delta y}\right]-k A\left[\frac{T(x, y)-T(x, y-1)}{\delta y}\right] \approx 0 .
\end{aligned}
$$


Obtaining the equations related to heat transfer by radiation is done using the band model, which subdivides the range of active wavelengths into finite bands within which the radiation properties are assumed grey [12, 13]. In the presented case, the band model separates solar and infrared bands, which results in the equations (3) and (4).

$$
\begin{gathered}
B_{S i}=\left(1-\varepsilon_{S i}\right) \sum_{j=1}^{n} \iint_{A_{j}} B_{S j} d F_{d A_{i} \rightarrow d A_{j}} . \\
B_{I i}=\varepsilon_{I i} \sigma T_{i}^{4}(x, y)+\left(1-\varepsilon_{I i}\right) \sum_{j=1}^{n} \iint_{A_{j}} B_{I j} d F_{d A_{i} \rightarrow d A_{j}}
\end{gathered}
$$

In Eq. (3) and Eq. (4), $d F_{d A_{i} \rightarrow d A_{j}}$ is known as the view factor, which is defined by the fraction of radiation leaving the surface $i$ that is intercepted by the surface $j$. The view factors for the surfaces in Fig. 3 were obtained analytically from the definition as presented in Sparrow and Cess [12].

Radiation heat flux on surface $S_{1}$ :

$$
q_{S i}^{\prime \prime}=\varepsilon_{I i} E_{1} \sigma T^{4}(x, y)-\varepsilon_{S} H_{S o l} E_{1} .
$$

Radiation heat flux on surfaces $S_{2}$ and $S_{3}$ :

$$
q_{R i}^{\prime \prime}=\frac{\varepsilon_{I i}\left[\sigma T_{i}^{4}(x, y)-B_{I_{i}}(x, y)\right]}{1-\varepsilon_{I i}}-\frac{\varepsilon_{S_{i}} B_{S_{i}}(x, y)}{1-\varepsilon_{S_{i}}} .
$$

The above equation represents the difference between the radiation emitted in the infrared band and the absorbed radiation in the solar band for each surface of the radiator. As can be seen, the term $T_{i}{ }^{4}$ is nonlinear. Such nonlinearity was solved using the Taylor series, as described by Shih [14],

$$
T^{4}(x, y)=4 T^{\prime 3}(x, y) T(x, y)-3 T^{\prime 3}(x, y)
$$

where $T^{\prime}(x, y)$ represents an initial estimate for the temperature.

The imposition of the described boundary conditions is done as follows. According to Fig. 3, we have:

1) For points on $S_{1}$ :

$$
k\left[\frac{T(x, y+1)-T(x, y)}{\delta y}\right]=4 \varepsilon_{I_{i}} \sigma T_{i}^{\prime}{ }^{3}(x, y) T_{i}(x, y)-3 \varepsilon_{I_{i}} \sigma T_{i}^{\prime}{ }^{3}(x, y)-\varepsilon_{S_{i}} H_{S o l} .
$$

2) $\quad$ For points on $S_{2}$ :

$k\left[\frac{T(x, y+1)-T(x, y)}{\delta y}\right]=-\frac{\varepsilon_{S_{i}} B_{S_{i}}(x, y)}{1-\varepsilon_{S_{i}}}+\frac{\varepsilon_{I_{i}}}{1-\varepsilon_{I_{i}}}\left\{\sigma\left[4 T^{\prime}{ }_{i}^{3}(x, y) T_{i}(x, y)-3 T^{\prime}{ }_{i}^{3}(x, y)\right]-B_{I_{i}}(x, y)\right\}$. 
3) For points on $S_{3}$ :

$k\left[\frac{T(x, y+1)-T(x, y)}{\delta y}\right]=-\frac{\varepsilon_{S_{i}} B_{S_{i}}(x, y)}{1-\varepsilon_{S_{i}}}+\frac{\varepsilon_{I_{i}}}{1-\varepsilon_{I_{i}}}\left\{\sigma\left[4 T^{\prime}{ }_{i}^{3}(x, y) T_{i}(x, y)-3 T^{\prime}{ }_{i}^{3}(x, y)\right]-B_{I_{i}}(x, y)\right\}$.

4) For points at $P_{1}$ :

$$
k\left[\frac{T(x+1, y)-T(x, y)}{\delta x / 2}\right]=0 .
$$

5) For points at $P_{2}$ :

$$
k\left[\frac{T(x, y)-T(x-1, y)}{\delta x / 2}\right]=0 .
$$

6) For points at $P_{E L}$ :

$$
k\left[\frac{T(x, y+1)-T(x, y)}{\delta y / 2}\right]=q_{E D}^{\prime \prime} .
$$

Dimensionless characteristic distances used:

$$
X^{\prime}=\frac{x}{D} ; \quad Y^{\prime}=\frac{y}{L}
$$

In order to perform a parametric analysis of the radiator and determine the best geometric characteristic parameters for heat dissipation, effectiveness parameter $\varepsilon$ was defined as the rate between the total heat flux obtained with the finned radiator and the total heat flux attained using the plan radiator in the same conditions, i.e.,

$$
\varepsilon \equiv q_{F R}^{\prime \prime} / q_{P R}^{\prime \prime}
$$

The model's validation has been performed by comparing results with those obtained using FlexPDE proprietary software for different conditions, and with those obtained by Garcia [8]. The grid analysis showed that no gain is obtained with a grid with more than 4000 elements, although the computational costs increase. 


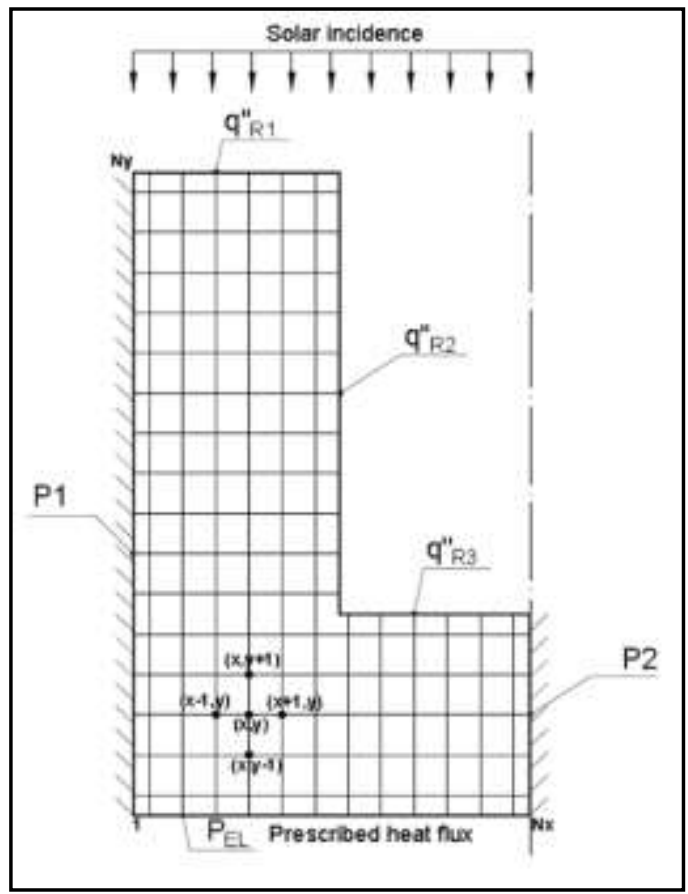

Fig. 3: Grid, boundary conditions and parameters.

\section{Numerical Solution}

The algorithm was developed using Fortran language, and it initializes by loading information such as thermo-optical parameters, view factors, the pseudo initial condition, boundary conditions and convergence criteria. Next, the program calculates the solar band radiosities, which do not depend on the surface temperatures. On the other hand, the radiosities in infrared band depend on the surface temperatures. Therefore, the pseudo initial conditions introduced at the initialization were used for such calculations. The calculated radiosities were then used to start the iterative process: the linear system of equations is solved using Thomas algorithm and then the new temperatures obtained override the previous temperatures and the process repeats until the difference between the temperatures of two consecutive iterations is less than the specified tolerance. After convergence is obtained, the radiation heat flux at all surfaces is calculated using 1/3 Simpson's rule as proposed by Campos [15]. In addition, radiosities leaving the surfaces towards the space are also calculated. Finally, in order to verify the results, the algorithm carries out the radiation energy balance as proposed by Garcia [8]. Fig. 4 outlines the algorithm's flowchart. 


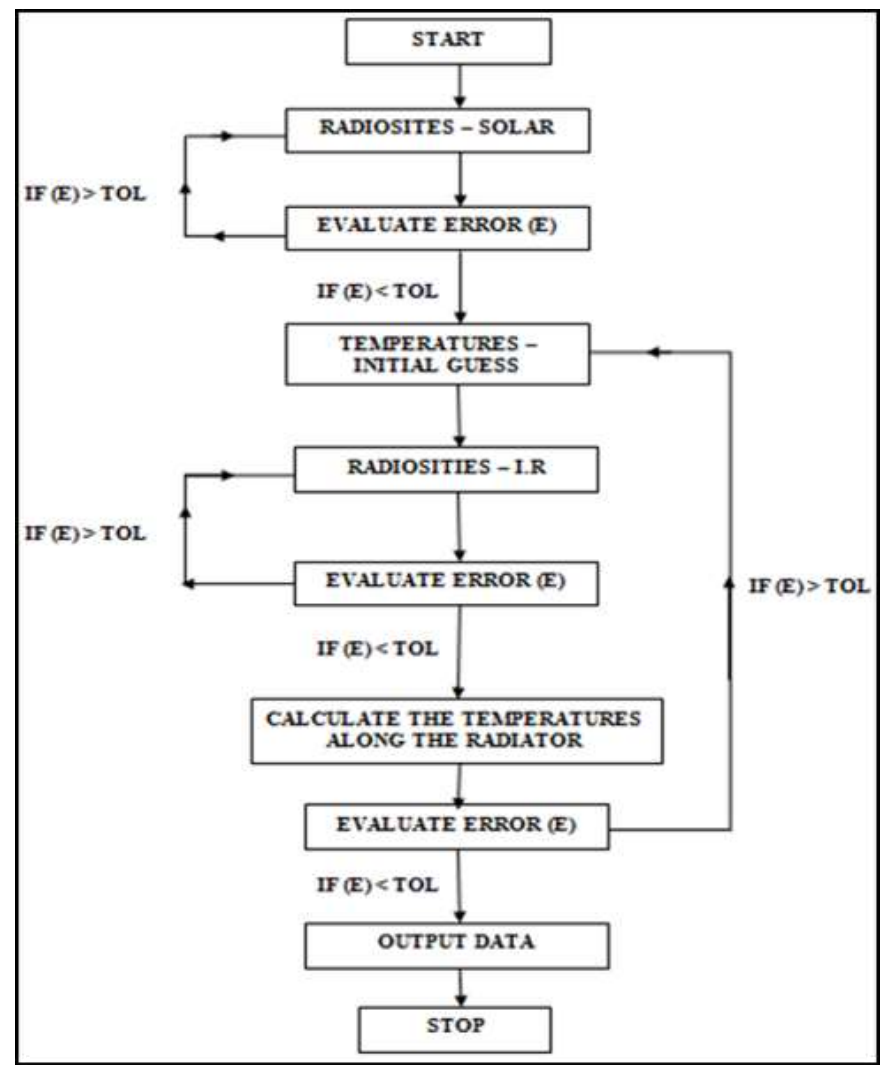

Fig. 4: Algorithm's flow chart.

\section{Results}

While the computational model determines the full temperature profile, results are only presented for the external surfaces since they have the strongest influence on radiator design by the fact that the radiation emitted by the radiator depends only on such temperatures. Fig. 5 presents the temperature profile on the surface $S_{2}$ for the hot case compared to an equivalent conventional flat plate radiator. Temperature gradients observed on $S_{2}$ demonstrate good performance of the fin radiator. The low gradients caused by the use of a high thermal conductivity surface and a thin radiator. Fig. 6 presents the temperature profile on the $S_{2}$ for the cold-case. The results show the same behavior obtained for the hot case, once again demonstrating the effectiveness of the finned radiator.

Figure 8 presents the effectiveness parameter $\varepsilon$ for different geometric configurations of the finned radiator in the same operation conditions. The results show that the geometrical configuration of the fins is a significant design factor. As can be seen in Fig. 8, the effectiveness decreases when the fins become too long. This can be explained by the fact that long fins will tend to induce a cavity effect between them by entrapping radiation leaving the radiator surfaces, while it has no effect on the incoming Sun irradiation. On the other hand, the results show that fins cannot be too short for the distance between then below $0.3 \mathrm{~m}$, otherwise its heat dissipation potential is not fully exploited. Finally, Fig. 8 indicates that the relation $L / D$ should not be superior to 0.5 in order to maximize the effectiveness.

For all the results presented, the specified tolerance was $10^{-10}$ and convergence was obtained. 


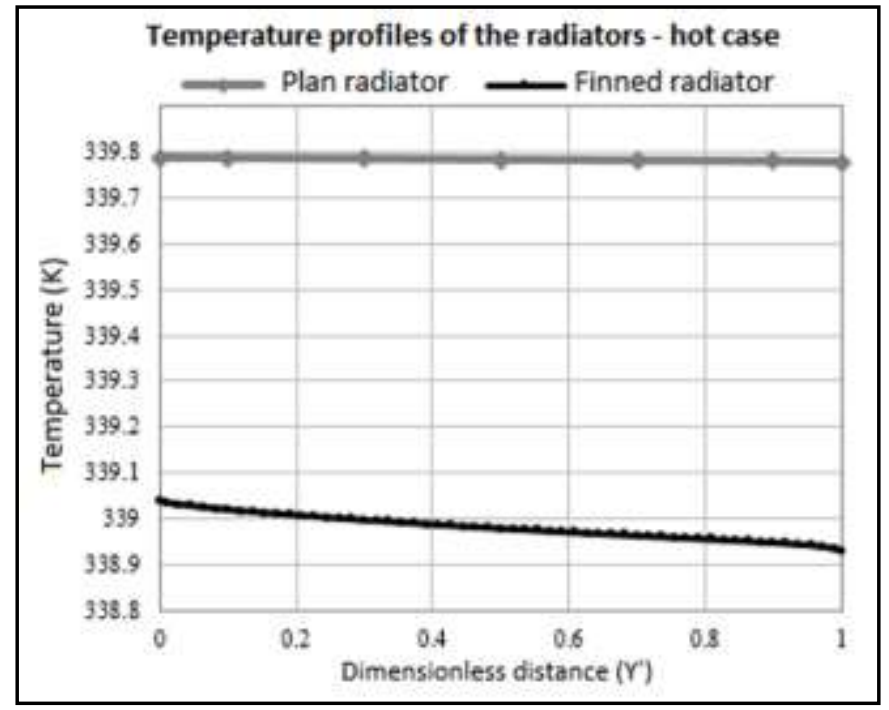

Fig. 5: Temperature profiles for the hot case.

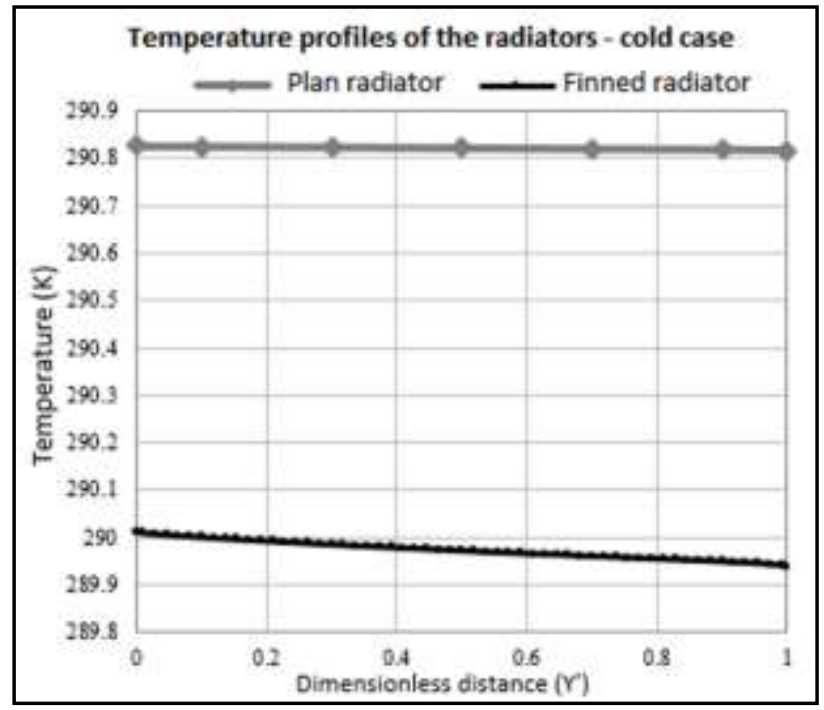

Fig. 6: Temperature profiles for the cold case.

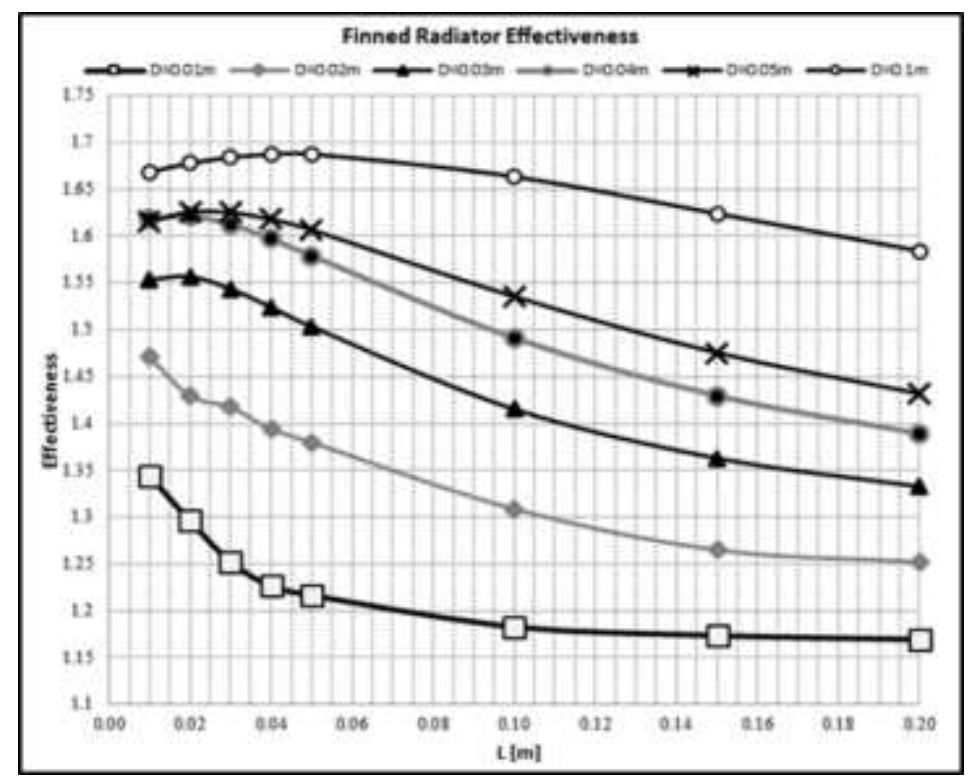

Fig. 7: Effectiveness parameter as a function of the fins`length and the spacing between the fins.

\section{Conclusions}

The work proposes a computational model for a finned space radiator to be used on a surface satellite aiming to increase its capacity of heat dissipation to the space, and therefore lowering the temperatures on the satellite. Two critical operational conditions were simulated. The two-dimensional approach did not provide very different results from those found with a one-dimensional approach presented by Garcia [8]. The fact that the no grid refinement was used near the walls introduced some divergences on the results to those obtained by the author. In the present work, it was bypassed with the use of a whole refined grid, which increased the computational costs. This suggests that for the analyzed geometries, the 1-D method proposed by Garcia [8] is reasonable. 
The results show that the finned radiator promotes a more effective heat exchange between the satellite's equipment and space. As a result, a smaller area of the satellite's surface is needed for heat dissipation purposes. It also provides the satellite's interior to be in appropriate conditions for the most critic equipment. Cold case analysis shows that even in the absence of solar radiation, the radiator does not compromise the satellite's operation due to radiator's high thermal conductivity. Therefore, the use of finned radiator for satellites' thermal control may can make heat pipes and heaters unnecessary. This fact, coupled with the low cost of production and the economy of area provided by the finned radiator demonstrate advantages of using such a device in the thermal control of satellites. Finned radiators are also mechanically reliable due to the absence of moving parts.

In future work, the use of adaptive-grid generation with parabolic partial differential equation as proposed by Noack and Anderson [16] should be investigated. A more realistic condition for the Sun irradiation will also be considered instead of the solar radiation focused perpendicularly to the base of the radiator.

\section{Acknowledgements}

The authors gratefully acknowledge the support for the present research provided by Fundação Coordenação de Aperfeiçoamento de Pessoal de Nível Superior, CAPES, through a doctoral scholarship under the Research Grant No. 88882.180821/2018-01 and through a sandwich doctoral scholarship under the Research Grant No. 88881.131976/2016-01 for the first author.

\section{Nomenclature}

$\begin{array}{ll}A & \text { cross-sectional area } \\ B & \text { radiosity } \\ \mathrm{D} & \text { distance between the fins } \\ d A & \text { infinitesimal area } \\ d F & \text { infinitesimal view factor } \\ E_{l} & \text { fin thickness } \\ E_{2} & \text { radiator's base thickness } \\ H_{S u n} & \text { normal incident solar radiation } \\ k & \text { thermal conductivity } \\ L & \text { fin length } \\ \mathrm{N} & \text { number of nodes } \\ q^{\prime \prime} & \text { heat flux } \\ q_{E D}^{\prime \prime} & \text { heat flux emanating from electronic device } \\ S & \text { surface } \\ T & \text { temperature } \\ X^{\prime} & \text { dimensionless horizontal distance } \\ Y^{\prime} & \text { dimensionless vertical distance } \\ x, y & \text { Cartesian coordinates } \\ \alpha & \text { absorptivity } \\ \delta & \text { finite distance } \\ \varepsilon & \text { effectiveness }\end{array}$




$\begin{array}{ll}\varepsilon_{i} & \text { emissivity (infrared band) } \\ \varepsilon_{S} & \text { emissivity (solar band) } \\ \sigma & \text { Stefan-Boltzmann constant } \\ \lambda & \text { wavelength } \\ \rho & \text { reflectivity } \\ \text { Subscript } & \\ E D & \text { electronic devices } \\ F R & \text { finned radiator } \\ I & \text { infrared band } \\ i, j & \text { generic finite volume } \\ P R & \text { plan radiator } \\ R & \text { radiative } \\ S & \text { Solar band } \\ \text { Superscript } \\ ()^{\prime} & \text { previous iteration } \\ ()^{\prime \prime} & \text { flux }\end{array}$

\section{References}

[1] A. F. S. Sorice, E. C. Garcia, I. Muraoka, K. E. de Conde, "Long-Term Evolution of SCD-1 Satellite Temperatures Based on a Comparative Analysis of Telemetric Data Measured in Orbit," Journal of Heat Transfer, vol. 138, 2016.

[2] D. F. Silva, "Análise de Projeto Preliminar de Controle Térmico do Satélite ITASAT," Masters thesis, Instituto Tecnológico de Aeronáutica - ITA, São José dos Campos, São Paulo, Brasil, 2009.

[3] A. Nazari, H. Emami, "Thermal Control and Thermal Sensors of Observation Satellite," The International Archives of Photogrammetry, Remote Sensing and Spatial Information Sciences, Beijing, 2008.

[4] S. M. Saboya, Análise Térmica de Coletor Solar com Absorvedor Aletado, Doctorate thesis, Instituto Tecnológico de Aeronáutica - ITA, São José dos Campos, São Paulo, Brasil, 1987.

[5] D. G. Gilmore, Spacecraft Thermal Control Handbook, Second Ed. The Aerospace Press, California, USA, 1994, vol. 1, $836 \mathrm{p}$.

[6] A. P. C. Cuco, F. L. Sousa, V. V. Vlassov, A. J. S. Neto, "Multi-Objetive Design Optimization of a New Space Radiator," Optimization and Engineering, vol. 12, no. 1, pp. 393-406, 2008.

[7] S. Lieblein, J. H. Diedrich, "Material and Geometry Aspects of Space Radiators," in Winter Meeting of the American Society, Washington D.C, USA, 1965.

[8] E. C. Garcia, Condução, Convecção e Radiação Acopladas em Coletores e Radiadores Solares. Doctorate thesis, Instituto Tecnológico de Aeronáutica - ITA, São José dos Campos, São Paulo, Brasil, 1996.

[9] D. F. Silva, E. C. Garcia, "Experimental and Numerical Investigation of Transverse Thermal Conductivity of an Aluminum Honeycomb Panel," International Review of Mechanical Engineering, vol. 8, pp. 344-349, 2014.

[10] S. S. K. Kumar, V. Nayak, S. P. Venkateshan, "Optimum Finned Space Radiators," International Journal of Heat and Fluid Flow, vol. 14, no. 2, pp. 191-200, 1993.

[11] R. R. Gasparin, Modelagem da Transferência de Calor Combinada por Condução e Radiação em Isolantes Térmicos de Edificações, Master thesis, Ponfifícia Universidade Católica do Paraná, Curitiba, Paraná, Brasil, 2005.

[12] R. S. E. M. Sparrow, R. D. Cess, Radiation Heat Transfer. MacGraw-Hill, New York, USA, 1978.

[13] R. Karam, "Satellite Thermal Control for Systems Engineers," American Institute of Aeronautics and Astronautics Inc., 
New York, USA, (1998).

[14] T. M. Shih, Numerical Heat Transfer, CRC Press, Hemisphere, Washington, USA, 1984.

[15] F. F. Campos, Algoritmos Numéricos. Second Ed. LTC Editora, Belo Horizonte, Minas Gerais, Brazil, 2007.

[16] R. W. Noack, D. A. Anderson, "Solution Adaptative Grid Generation Using Parabolic Partial Differential Equations," AIAA 26th Aerospace Sciences Meeting, Reno, Nevada, USA, 1990. 